\title{
Increased pulse repetition frequency for effective and less painful electrochemotherapy
}

\author{
D. Miklavčič' , G. Pucihar' ${ }^{1}$, L. M. Mir ${ }^{2}$, M. Puc ${ }^{1}$, K. Flisar ${ }^{1}$, \\ A. Maček Lebar ${ }^{1}$, M. Mali ${ }^{1}$, G. Serša ${ }^{3}$, S. Kranjc ${ }^{3}$, M. Čemažar ${ }^{3}$, \\ M. Petkovšek ${ }^{1} \&$ J. Nastran ${ }^{1}$

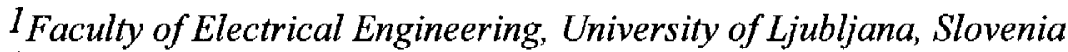 \\ 2 UMR 8532 CNRS, Institute Gustave-Roussy, France \\ 3 Department of tumor biology, Institut of Oncology, Slovenia
}

\begin{abstract}
Electrochemotherapy is a technique where electric pulses in combination with chemotherapeutic agents are applied to tumor cells. In general, patients find electrochemotherapy tolerable, in spite of unpleasant sensations associated with contraction of muscles located in the vicinity of the electrodes. Usually a train of eight electric pulses with repetition frequency of $1 \mathrm{~Hz}$ is applied to the tumors and each pulse in the train provokes muscle contraction. The use of pulses with repetition frequency higher than the frequency of tetanic contraction would represent reduced number of muscle contractions and unpleasant sensations. To study the possibilities for the use of higher pulse repetition frequencies in ECT, the effect of increased pulse repetition frequency on the uptake of Lucifer Yellow in vitro, the effect of increased pulse repetition frequency on the tumor growth after ECT in vivo and the effect of different pulse repetition frequencies on the muscle response in healthy volunteers were investigated. In vitro results showed that the uptake of Lucifer Yellow stays at similar levels at frequencies up to $1 \mathrm{kHz}$. In vivo experiments demonstrated that ECT with higher pulse repetition frequencies is still effective. Muscle response measured at different pulse repetition frequencies confirmed that the use of pulse repetition frequencies above the frequency of tetanic contraction reduces the number of individual muscle contractions and further increase of the pulse repetition frequency results in decreased muscle response due to shorter total duration of pulse train.
\end{abstract}




\section{Introduction}

The combined local treatment consisting of a nonpermeant anticancer drug possessing a high intrinsic cytotoxicity (e.g. bleomycin, cisplatin) and short, high voltage electric pulses has been termed electrochemotherapy (ECT). At the appropriate amplitude of the pulses the permeability of tumor cell membranes increases transiently, therefore allowing the chemotherapeutic drugs to enter the cell. ECT is typically administered by injecting a drug, systemically or locally, and then applying pulses directly to the tumor after allowing time for the drug to disseminate throughout the treatment site. Outstanding antitumor effects have been reported in preclinical studies in a variety of cutaneous and subcutaneous tumors. In the most comprehensive study [1], the results of five different research groups (France, USA and Slovenia) were summarized. Objective responses (absence of any trace of tumor, complete response, or more than $50 \%$ reduction in tumor volume, partial response, for at least 30 days after the treatment) were obtained in $85.3 \%$ of the 273 tumors evaluated, regardless of the histological type of the treated tumors.

Treatment of tumors in these studies included six or eight rectangular direct current pulses, $100 \mu$ s of duration with repetition frequency of $1 \mathrm{~Hz}$. In general, the patients found ECT tolerable, although some complained about unpleasant sensations associated with the delivery of electric pulses. Because of the relatively low repetition frequency of the pulses, each individual pulse excites the nerve fibers located below and in the vicinity of the electrodes and provokes the contraction of muscles innervated by the excited neurons. Consecutive muscle contractions during ECT are painful and unpleasant for the patients; therefore any reduction of these sensations would be a great improvement of the promising therapy.

The use of pulses with repetition frequency exceeding the frequency of tetanic contraction (where successive muscle contractions fuse into smooth motion) would already represent an improvement in sense of reducing the pain associated with ECT due to reduced number of consecutive muscle contractions but would also increase the force of muscle contraction (Figure 1) [2]. If the pulses are delivered at the frequency for which the duration of the whole train of pulses is shorter than duration of the action potential with the refractory period (Figure 2), the force of muscle contraction and the number of muscle contractions would probably be reduced considering finite duration of the train of pulses (filled circles in Figure 1). For example, eight $100 \mu$ s pulses would provoke a single action potential and thereby a single muscle contraction if the repetition frequency of the pulses is in the range of a few $\mathrm{kHz}$; the total duration of a pulse train at $1 \mathrm{~Hz}$ repetition frequency is $8 \mathrm{~s}$, while at $1 \mathrm{kHz}$ pulse repetition frequency the total duration of the train is $8 \mathrm{~ms}$. 


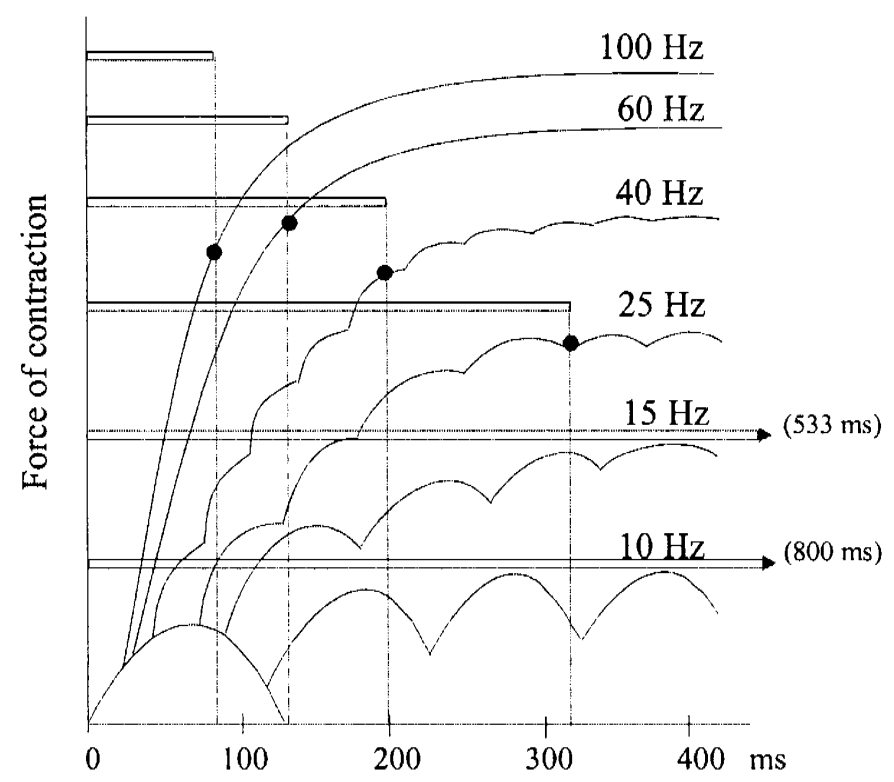

Figure 1. The dependence of the force of muscle contraction on the pulse repetition frequency and the occurrence of tetanic contraction for continuous muscle stimulation. The symbols $(\bullet)$ represent the force of contraction due to excitation with a pulse train $(8 \times 100 \mu \mathrm{s})$ at a given frequency.

duration of a pulse train $(8 \times 100 \mu \mathrm{s})$ at a given repetition

To study the possibilities for the use of higher pulse repetition frequencies in ECT, first the effect of increased pulse repetition frequency on the uptake of Lucifer Yellow in vitro was investigated. Second, we followed the effect of increased pulse repetition frequency on the tumor growth after ECT in vivo. And finally, we studied the effect of different pulse repetition frequencies on the muscle response in healthy volunteers.

\section{Methods}

\subsection{In vitro}

DC3F cells were grown in Eagle's Minimum Essential Medium (EMEM) with added $10 \%$ Fetal bovine serum (both from Life Technologies, USA). After trypsination, cells were centrifuged for $5 \mathrm{~min}$ at $1000 \mathrm{rpm}$ at $4{ }^{\circ} \mathrm{C}$ and resuspended in Spinner's minimum essential medium (SMEM, Life Technologies, USA) to obtain $2 \times 10^{7}$ cells $/ \mathrm{ml}$. 


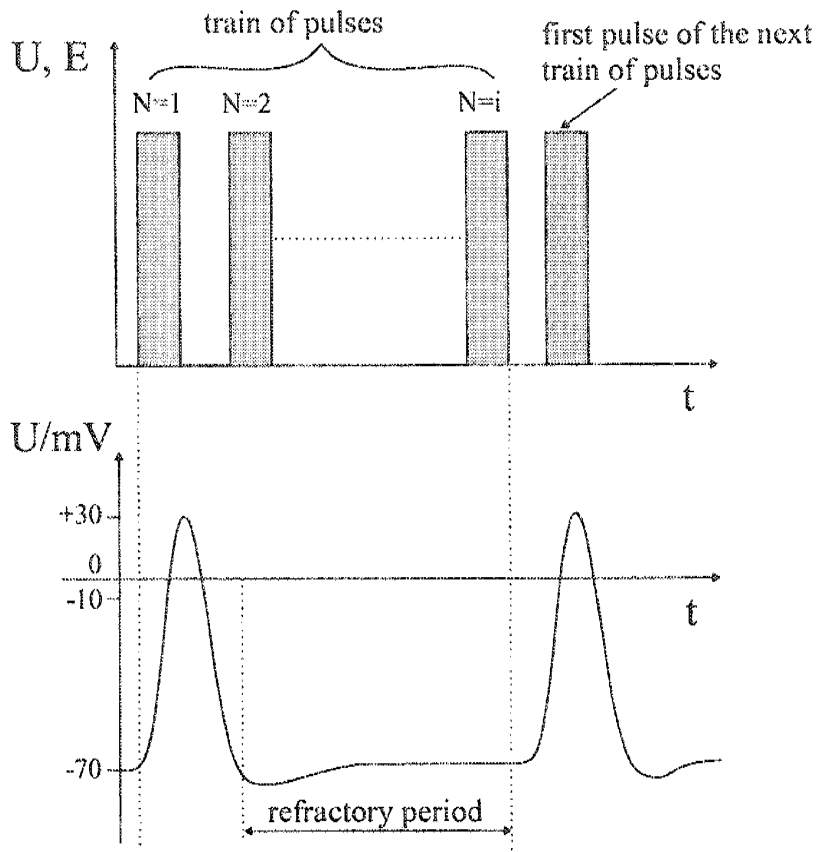

Figure 2. The duration of the whole train of pulses in comparison with the duration of the action potential with its refractory period.

To obtain the uptake of molecules into permeabilized cells, a nonpermeant fluorescence dye Lucifer Yellow (LY) was added to cell suspension before electropermeabilization in quantity that leads to $1 \mathrm{mM}$ concentration of LY in cell suspension. Cells were kept at $4{ }^{\circ} \mathrm{C}$ until electropermeabilization.

A $50 \mathrm{ml}$ droplet of cell suspension $\left(10^{6}\right.$ cells $)$ was put between two parallel plate stainless steel electrodes $2 \mathrm{~mm}$ apart. Eight rectangular pulses, duration 100 $\mu \mathrm{s}$, with amplitudes from $80 \mathrm{~V}$ to $400 \mathrm{~V}$, and repetition frequencies $1 \mathrm{~Hz}, 10 \mathrm{~Hz}$, $100 \mathrm{~Hz}$, and $1 \mathrm{kHz}$ were delivered. After incubation $\left(10 \mathrm{~min}, 25^{\circ} \mathrm{C}\right), 950 \mathrm{ml}$ of SMEM was added to prevent drying.

After additional $30 \mathrm{~min}$, cells were diluted in $5 \mathrm{ml}$ of PBS (Life Technologies, USA) in order to wash extracellular LY and centrifuged at $1000 \mathrm{rpm}$. Cells were then ultrasonicated (Sonifer 250, Branson Ultrasonics, USA) and fluorescence was measured on a spectrofluorometer (SFM 25, BioTek, USA). Excitation was set at $418-\mathrm{nm}$ wavelength and emission was detected at $525 \mathrm{~nm}$. The background fluorescence was subtracted, and the fluorescence was converted into intracellular concentration of LY. 
All experiments were repeated at least three times. Results from different repetitions of experiments were pooled together and are presented as the mean and standard deviation of the mean.

\subsection{In vivo}

In the experiments, the inbred $\mathrm{A} / \mathrm{J}$ mice of both sexes were used, which were purchased from Rudjer Boškovič Institute (Zagreb, Croatia). They were maintained at a constant room temperature $\left(24^{\circ} \mathrm{C}\right)$ with a natural day/night cycle in a conventional animal colony. Before the experiments, the mice were subjected to an adaptation period of at least 10 days. Mice in good condition, without fungal or other infections, weighing 20-22g and 10-12 weeks old were included in experiments.

The fibrosarcoma SA-1 tumor model (The Jackson Laboratory, Bar Harbor, USA), syngeneic to $\mathrm{A} / \mathrm{J}$ mice, was used in the study. Tumor cells were obtained from the ascitic form of the tumors in mice, serially transplanted every 7 days. Solid subcutaneous tumors, located dorsolaterally in mice, were initiated by an injection of $5 \times 10^{5} \mathrm{SA}-1$ cells in $0.1 \mathrm{ml} 0.9 \% \mathrm{NaCl}$ solution. The viability of the cells, as determined by a trypan blue dye exclusion test, was over $95 \%$. Six days after transplantation, when the tumors reached approximately $40 \mathrm{~mm}^{3}$ in volume, mice were randomly divided into eight experimental groups, and subjected to a specific experimental protocol on day 0 .

Bleomycin at a dose of $50 \mathrm{mg}$ per animal was injected intravenously. Electric pulses were delivered by two flat, parallel stainless steel plate electrodes with rounded comers (length $=35 \mathrm{~mm}$, width $=7 \mathrm{~mm}$, thickness $=1 \mathrm{~mm}$, interelectrode distance $=8 \mathrm{~mm}$ ). They were placed at the opposite margins of the tumor in cranial/caudal direction. Good contact between the electrodes and the skin was assured by means of conductive gel. Eight $100 \mu$ s monophasic squarewave electric pulses with pulse amplitude of $1040 \mathrm{~V}$ and repetition frequencies 1 $\mathrm{Hz}, 10 \mathrm{~Hz}, 100 \mathrm{~Hz}, 1 \mathrm{kHz}$ and $5 \mathrm{kHz}$ were generated by electropulsator, which was developed at the University of Ljubljana [3]. In the combined treatment groups, mice were treated with electric pulses $3 \mathrm{~min}$ after BLM injection, which is sufficient for the distribution of BLM. Mice in the control and electric pulses only groups were injected with 0.01 M PBS (pH 7.4) instead of bleomycin. Experiment was repeated twice.

Tumor growth was followed by measuring three mutually orthogonal tumor diameters (e1, e2 and e3) with vernier caliper on each consecutive day. Tumor volumes were calculated by the formula $V=\pi \times e_{1} \times e_{2} \times e_{3} / 6$. From the measurements, the arithmetic mean and standard error of the mean were calculated for each experimental group. If the tumor became unpalpable and did not regrow after 100 days, the therapeutic response was scored as complete response (CR). 


\subsection{Muscle response}

Ten healthy volunteers of both sexes, age 20 to 24 years, were included in the study. Subjects were seated in a comfortable chair with the knee flexed at 90 degrees. The ankle joint was aligned with the axis of rotation of the ankle joint torque meter (Jožef Stefan Institute, Ljubljana, Slovenia) consisting a straingauge transducer that transformed torque into a voltage. Then the foot and lower limb were firmly fixed.

An isometric twitch contraction was elicited by stimulating the tibialis anterior muscle with eight $100 \mu$ s square wave pulses, with amplitude of $90 \mathrm{~V}$ and repetition frequencies of $1,10,50,100,500,1000$ and $1250 \mathrm{~Hz}$. The skin was slightly abraded and cleaned with ethyl alcohol. Bipolar, self-adhesive surface electrodes were placed on the skin, one on the motor point and the other near the tendon. We used a GRASS S88 stimulator (Grass Product Group, Astro-Med, Inc., West Warwick, USA), with voltage output, in conjunction with an insulation unit. The measured muscle responses were stored to a computer and analyzed using Matlab 5.3 Software (Math Works, Inc., Natick, USA).

\section{Results}

\subsection{In vitro}

The results of the uptake of Lucifer Yellow into electropermeabilized cells as a function of pulse amplitude and repetition frequency of electric pulses are shown in Figure 3. As the figure shows, increasing the amplitude of the pulses results in

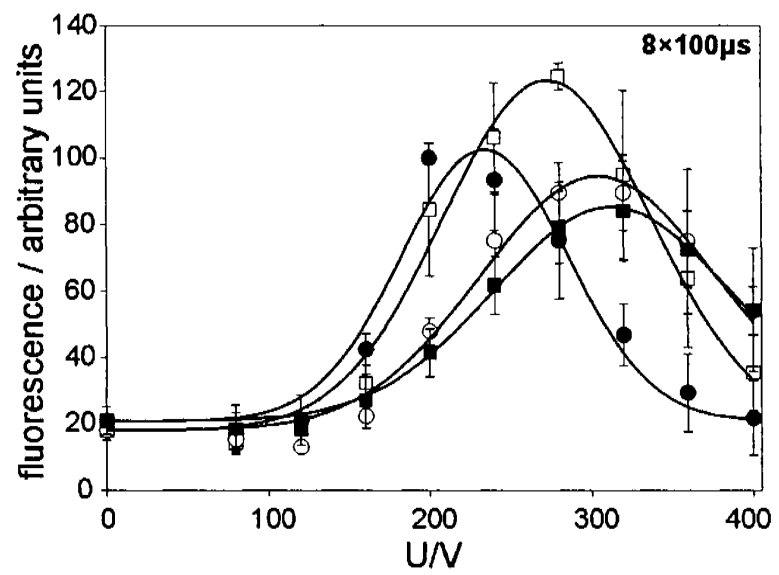

Figure 3. The uptake of LuciferYellow (LY) as a function of pulse amplitude U at pulse repetition frequencies of $1 \mathrm{~Hz}(\bullet), 10 \mathrm{~Hz}(\square), 100 \mathrm{~Hz}(O)$ and $1 \mathrm{kHz}(\square)$ (8 pulses of $100-\mu$ s duration). The results are represented as the mean and standard deviation of the mean. 
an increase of the uptake of Lucifer Yellow, which reaches its maximum value at a certain pulse amplitude. With further increase of the pulse amplitude the uptake decreases as a result of irreversible cell electropermeabilization. The highest uptake was obtained at the frequency of $10 \mathrm{~Hz}$. Even at the highest pulse repetition frequency examined $(1 \mathrm{kHz})$, the uptake is not reduced significantly. However, higher pulse amplitudes seem to be required to obtain the maximum uptake with an increase of the repetition frequency of electric pulses.

\subsection{In vivo}

Electrochemotherapy with all five repetition frequencies of electric pulses induced an arrest of tumor growth (Figure 4). It can be seen, that the effectiveness of ECT at different pulse repetition frequencies is comparable.

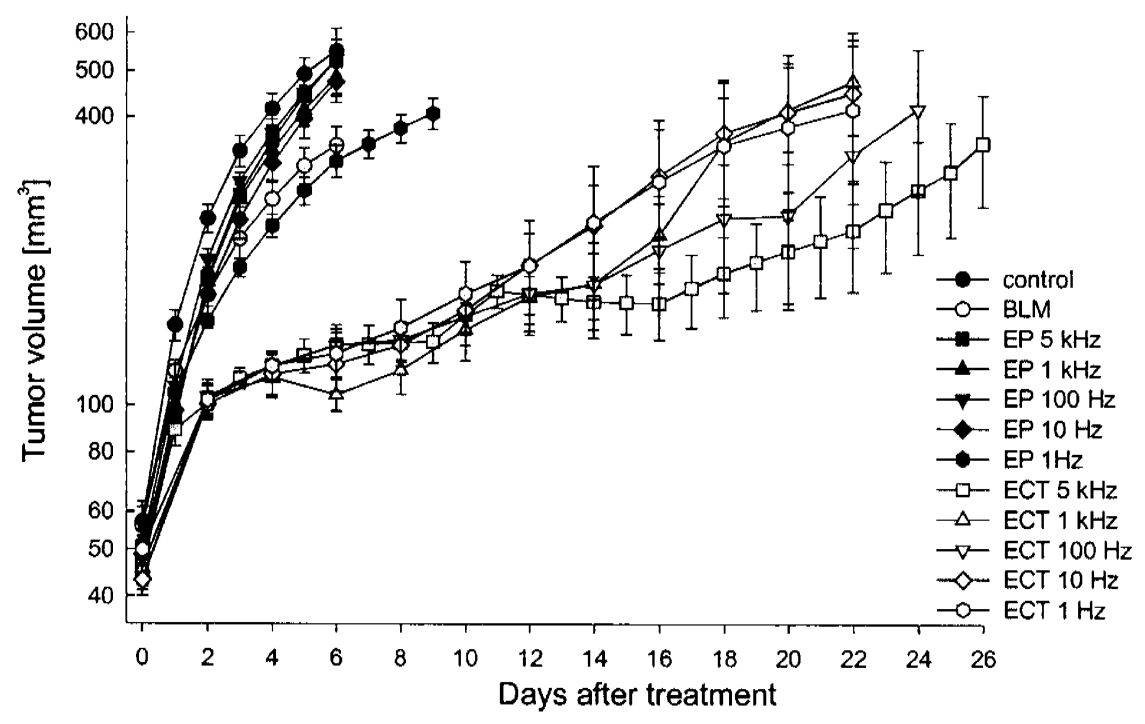

Figure 4. Tumor growth curves. The results are presented as mean and standard error of the mean.

The antitumor effectiveness of ECT with different pulse repetition frequencies is also shown in the percentage of CR (Table 1). The highest percentage of CR $(36.8 \%)$ was observed for the "standard" repetition frequency $1 \mathrm{~Hz}$, and the lowest $(9.1 \%)$ for repetition frequency $1 \mathrm{kHz}$. However, differences were not statistically significant. 
Table 1. Antitumor effectiveness of ECT with different pulse repetition frequencies. $\mathrm{N}$ - number of animals in each experimental group; CRnumber of complete responses.

\begin{tabular}{|l|l|l|}
\hline Therapy & N & CR [\%] (CR) \\
\hline control & 16 & $0(0)$ \\
\hline BLM & 12 & $0(0)$ \\
\hline EP $1 \mathrm{~Hz}$ & 10 & $0(0)$ \\
\hline EP $10 \mathrm{~Hz}$ & 13 & $0(0)$ \\
\hline EP $100 \mathrm{~Hz}$ & 12 & $0(0)$ \\
\hline EP $1 \mathrm{kHz}$ & 7 & $0(0)$ \\
\hline EP $5 \mathrm{kHz}$ & 13 & $0(0)$ \\
\hline ECT $1 \mathrm{~Hz}$ & 19 & $36.8(7)$ \\
\hline ECT $10 \mathrm{~Hz}$ & 18 & $11.1(2)$ \\
\hline ECT $100 \mathrm{~Hz}$ & 17 & $29.4(5)$ \\
\hline ECT $1 \mathrm{kHz}$ & 11 & $9.1(1)$ \\
\hline ECT $5 \mathrm{kHz}$ & 18 & $22.2(4)$ \\
\hline
\end{tabular}

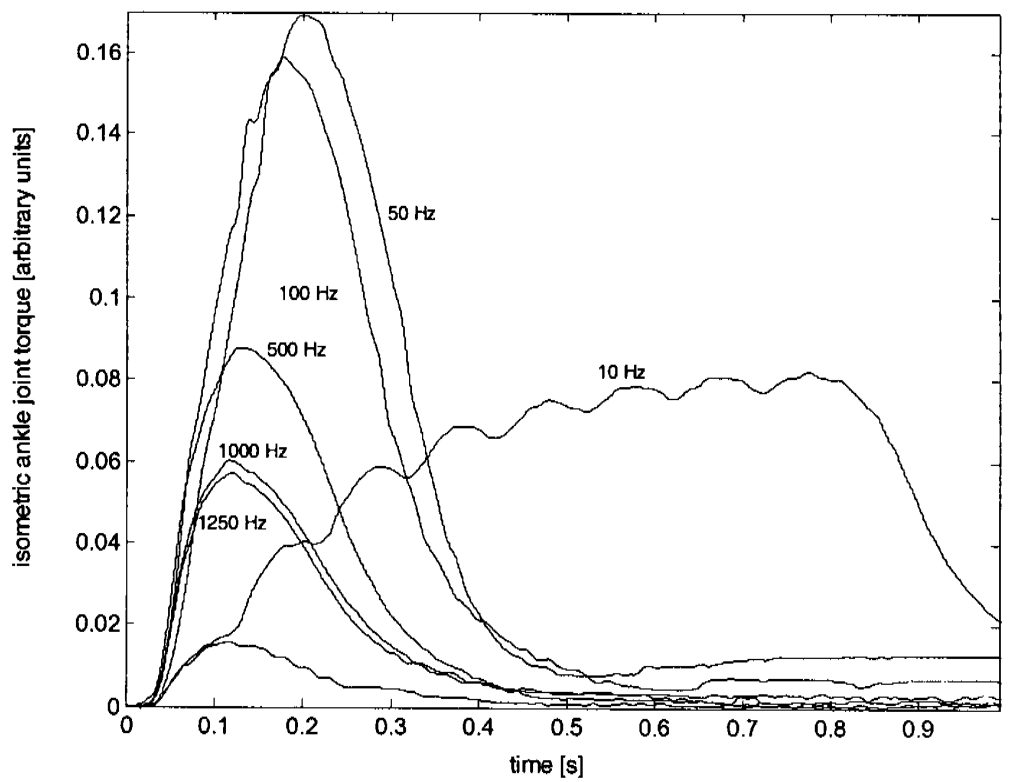

Figure 5. The time dependence of isometric ankle joint torque. Stimulation frequencies were $1,10,50,100,500,1000,1250 \mathrm{~Hz}$. 


\subsection{Muscle response}

The isometric ankle joint torque as a function of time and the repetition frequency of electric pulses is shown in Figure 5. The train of pulses with repetition frequency just above the frequency of tetanic contraction $(50 \mathrm{~Hz})$ reduces the number of muscle contractions to one contraction, but the muscle response is high. At higher frequencies $(500,1000$ and $1250 \mathrm{~Hz})$ the muscle response decreases.

\section{Conclusions}

The purpose of our study was to examine the possibilities of using pulses with repetition frequencies higher than $1 \mathrm{~Hz}$ in ECT. In vitro results showed that the uptake of Lucifer Yellow at highest repetition frequency examined $(1 \mathrm{kHz})$ stays at similar levels as the uptake at $1 \mathrm{~Hz}$. The results from in vivo experiments demonstrated that ECT with higher pulse repetition frequencies is still effective. Muscle response measured at different pulse repetition frequencies confirmed our theoretical considerations; the use of pulse repetition frequencies above the frequency of tetanic contraction reduces the number of individual muscle contractions and further increase of the pulse repetition frequency eventually results in decreased muscle response due to shorter total duration of pulse train.

In summary, we suggest the use of pulses with higher frequencies as an improvement of existing ECT in a sense of reduced number of muscle contractions and decreased muscle response.

\section{Acknowledgements}

This work was supported by the Ministry of Education, Science and Sport of the Republic of Slovenia, the Proteus program of scientific, technological and cultural co-operation between France and Slovenia and the Cliniporator project (Grant QLK3-1999-00484) from the European Commission. Part of this research was also supported by IGEA Carpi (Modena), Italy.

\section{References}

[1] Mir, L.M., Glass, L.F., Serša, G., Teissie, J., Domenge, C., Miklavčič, D., Jaroszeski, M.J., Orlowski, S., Reintgen, D.S., Rudolf, Z., Belehradek, M., Gilbert, R., Rols, M.P., Belehradek Jr., J., Bachaud, J.M., DeConti, R., Štabuc, B., Čemažar, M., Coninx, P. \& Heller, R., Effective treatment of cutaneous and subcutaneous malignant tumors by electrochemotherapy. $B r . J$. Cancer, 77, pp. 2336-2342, 1998.

[2] Pucihar, G., Mir, L.M. \& Miklavčič, D., The effect of pulse repetition frequency on the uptake into electropermeabilized cells in vitro with possible applications in electrochemotherapy. Bioelectrochemistry, 57, pp. 167-172, 2002. 
400 Simulations in Biomedicine $V$

[3] Petkovšek, M., Nastran, J., Vončina, D., Zajec, P., Miklavčič, D. \& Serša, G., High voltage pulse generation. Electronics letters, 38, pp. 680-682, 2002. 